\title{
The Determination of Complete Pole Figures Using the Reflection Method
}

\author{
FAN XIONG + and B. A. PARKER \\ Department of Materials Engineering, Monash University, Clayton, Victoria 3168, \\ Australia
}

†On leave from Tianjin University, Tianjin, China

(Received May 22, 1984; in final form July 19, 1984)

\begin{abstract}
A method is described which enables complete pole figures of metal sheet to be obtained using the reflection technique alone. A small known displacement of the sample from the centre of the texture goniometer is made and this significantly reduces absorption at high tilt angles (the outer region of the pole figure). The displacement introduced requires a wider receiving slit in order to collect an adequate fraction of the diffracted $\mathrm{x}$-rays. The overall improvement in peak-to-background ratio is such that reproducible, complete pole figures can be produced. No loss in the detail contained in the pole figures has been observed due to the misalignment of the goniometer geometry.
\end{abstract}

\section{INTRODUCTION}

The reflection goniometer originally proposed by Schultz (1949) is the most common instrument used for obtaining pole figures describing the preferred orientation of the grain structure in metal sheets. A limiting feature of the instrument is that there is inadequate diffracted intensity when the sample is inclined such that the sheet normal approaches an angle of $90^{\circ}$ with the plane containing the incident and diffracted beams ( $\phi$ in the Schultz geometry, Figure 1). The limit is due to strong absorption of the $\mathrm{x}$-ray beam in the sample and is in the range $75-80^{\circ}$ for typical samples and experimental configurations. 


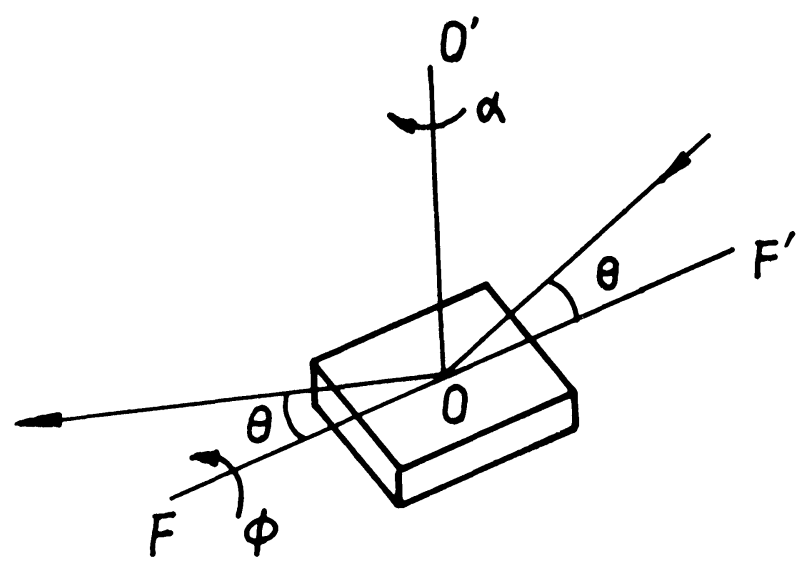

FIGURE 1 Sample/goniometer geometry in the Schultz reflection method. The Bragg angle $\theta$ is fixed and the sample rotates about the sheet normal $00^{\prime}(\alpha)$ and tilts about $\mathrm{FF}^{\prime}(\phi)$.

Alternative methods for obtaining complete pole figures include the following. (i) Combining both reflection and transmission figures $(\mathrm{Hu}$ et al., 1952), this can be time consuming and matching the figures may be difficult if the texture varies through the thickness of the sample. (ii) Making a composite specimen of several thicknesses of sheet and cutting it so that a complete quadrant of the pole is obtained in reflection (Lopata and Kula, 1962). The pole figure obtained is an average through the sheet thickness and also assumes symmetry of the pole figure. (iii) Making a composite specimen as in (ii), cutting a small spherical specimen from it and the pole figure determined using transmission geometry (Jetter and Borie, 1953). This technique has the advantage that absorption corrections are small. However, specimen preparation is time consuming and, again, an average through the sheet thickness is obtained. The averaging may be desirable for some applications but it may also be necessary to know how the texture varies through the sheet, and this is difficult to discern using the composite specimen methods.

A somewhat similar approach to that described in this paper has been adopted, recently, by Ortiz and Hermida (1981) who derived a theoretical expression for the effect of defocussing on the diffracted intensity. Their work was based on a number of earlier treatments Chernock and Beck, 1952; Tenckhoff, 1970; Feng, 1965). Precise experi- 
mental details were not given and the application of their expression requires a measurement of the misalignment for each change in geometry. They have published a further paper (Ortiz and Hermida, 1980) which describes a method for testing the goniometer alignment.

\section{PRINCIPLE OF THE METHOD}

The geometry of the Schultz reflection method is shown in Figure 1. The incident and reflected beams are shown at a Bragg angle $\theta$ to the sheet specimen. The angle $\alpha$ describes the rotation of the sample about an axis which is the sheet normal and the angle $\phi$ represents the tilting of the sample plane with respect to the plane containing the incident

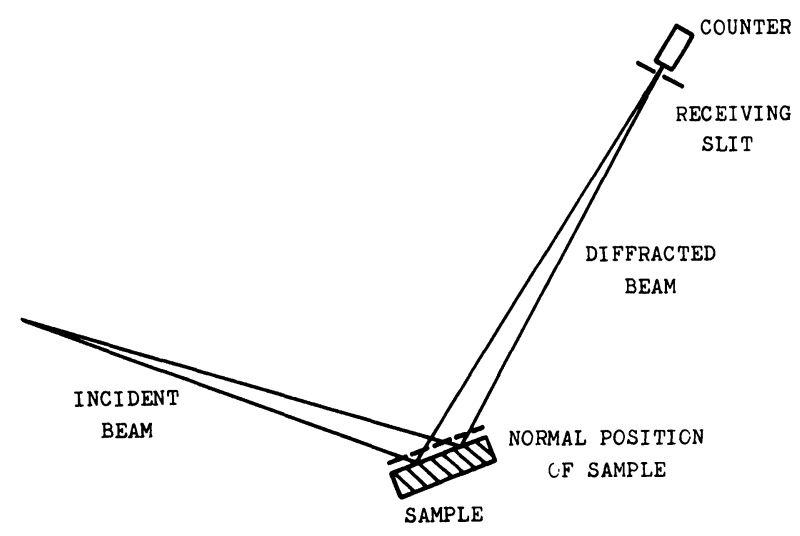

(a)

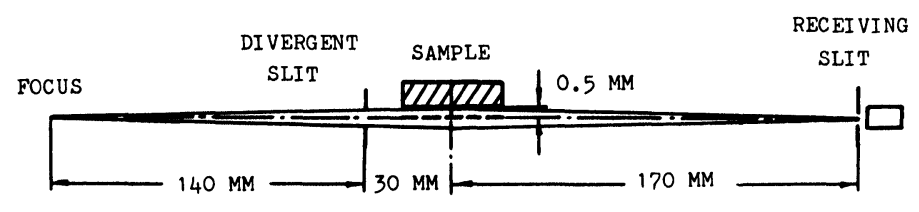

(b)

FIGURE 2 Beam geometry in the experimental arrangement described.

a) side view, $\phi=0^{\circ}$.

b) top view, $\phi=90^{\circ}$. 
and diffracted beams. High absorption of $\mathrm{x}$-rays occurs at high $\phi$ angles and this can be appreciated from the experimental geometry.

It may be seen that the amount of absorption will be reduced if the sample is displaced along $00^{\prime}$ such that the centre of the Rowland circle lies above the sample. This misalignment causes defocussing but, as will be seen from the results, the defocussed beam can be collected using a wide receiving slit and useful intensities can be obtained for the full range of $\phi$ angles $\left(0-90^{\circ}\right)$. The intensity arises because the incident $x$-ray beam is not parallel but diverges from the collimator. Part of the divergent beam strikes the sample in its displaced position as shown in Figure 2. The fraction of the beam striking the specimen is a function of the angle $\phi$ and may be calculated from a knowledge of the goniometer geometry and the profile of the incident beam. Experimentally, however, it is more convenient to make use of the additional intensity and make the necessary corrections for absorption and defocussing using a random sample. The optimum amount of specimen displacement to maximise the available intensity at $\phi=90^{\circ}$ is a function of the collimator/sample/goniometer geometry and the experimental section includes a determination of the optimum value for our present installation.

The results section demonstrates that the pole figures obtained using the modified geometry remain essentially unchanged (except for a known change in the absorption correction) and may be used directly or as an input to an ODF analysis.

\section{EXPERIMENTAL ARRANGEMENT}

The apparatus comprises a Philips PW1078 texture goniometer mounted on a $1 \mathrm{kw}, 0.1 \%$ stabilized $\mathrm{x}$-ray generator. The goniometer is continuously scanned over the $\phi$ and $\alpha$ angles and data collection is achieved using an Apple microcomputer, using a peripheral card incorporating programmable counter/timers. A pulse height analyzer is incorporated between the proportional detector and the interface. Each measurement represents an average intensity over a small angular interval.

Two modifications have been made to the standard goniometer. The most important change is the displacement of the sample surface noted in the introduction. In order to find an optimum value for this displacement, several known displacements were made and the profile 
of the Bragg peaks and, also, complete pole figures determined. The results are noted later.

The second modification involved the widening of the receiving slit in order to collect a greater proportion of the diffracted $\mathrm{x}$-rays since a result of defocusing is that the angular spread of the diffracted $x$-rays is greater than with the normal geometry. There is also a small shift in the $2 \theta$ value of the peak maximum since the fraction of the incident beam used at high $\phi$ angles only strikes the sample due to its divergence from the true $\theta$ value (see Figure 2).

The modifications allowed adequate counting statistics at all $\phi$ angles using a $1 \mathrm{kw}$ copper tube and a 4 second counting time. The $\alpha$ angle varied $3^{\circ}$ during each measurement and the pitch of the spiral was $5^{\circ}$, thus generating 2160 data points for a complete pole figure. The resolution of detail in the pole figures is adequate for most purposes and is, at present, being evaluated as input to ODF programmes. The quality of the pole figures was not affected, for practical purposes, by defocusing and the results are indistinguishable from those obtained by other methods. Typical pole figures are included in the results section.

Intensity correction for defocusing and absorption was achieved with the aid of a sample made by loading aluminium powder into a small quantity of epoxy resin. This 'random' sample showed no preferred orientation and the intensity profile obtained, as a function of the angle $\phi$ was used to correct the intensity obtained from textured specimens prior to plotting the pole figure.

\section{RESULTS}

\section{Receiving slit width}

Figure 3 shows the normalised intensity of the (111) reflection from the random sample as a function of $\phi$ for (a) the slit configuration supplied with the instrument, with the specimen at its correct position; (b) as for (a) but with a receiving slit width of $4 \mathrm{~mm}$ (note that the intensity is now constant out to $\phi=45^{\circ}$ ), and (c) with the wide receiving slit and the optimum sample displacement described below. The intensity in (c) is almost constant out to $\phi=70^{\circ}$ and useful intensities are obtained at $\phi=90^{\circ}$. The effects of the reduced intensity incident on the specimen and the absorption of the diffracted beam are shown in 

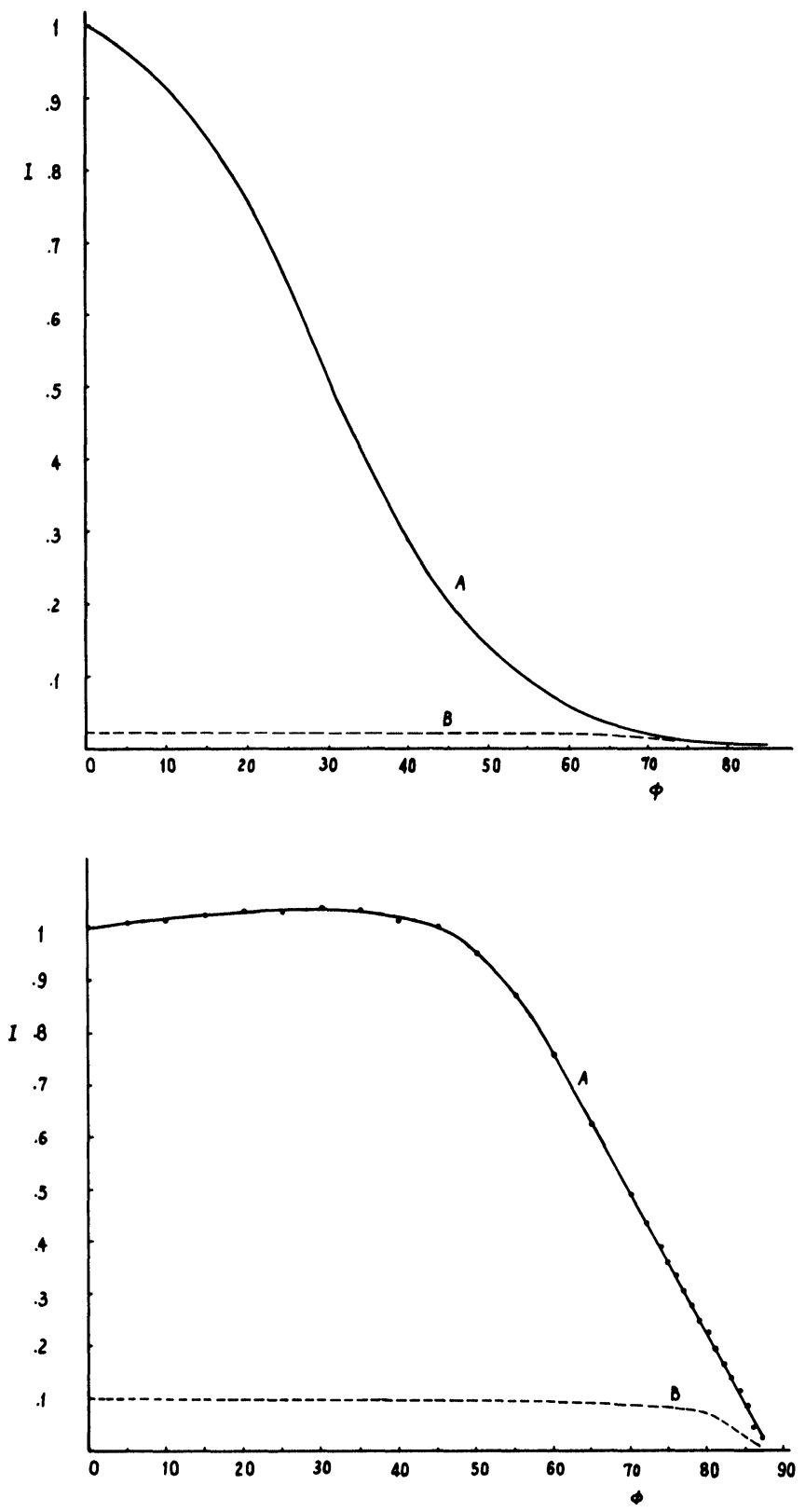


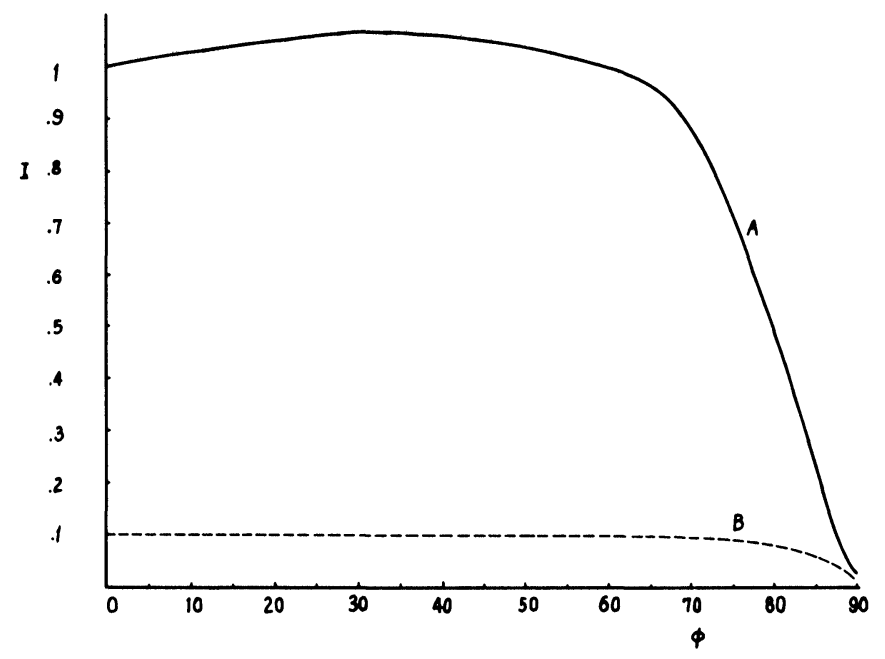

FIGURE 3 Diffracted intensity as a function of the tilt angle $\phi$. A. total intensity; B. background intensity.

a) Standard geometry, narrow receiving slit.

b) Standard geometry, wide receiving slit.

c) As (b) but with optimum sample displacement (see text).

the change of background intensity with the angle $\phi$ measured with the goniometer well off the Bragg position.

It should be noted here that the slits originally supplied with the goniometer were not an optimum for texture determination, even with the standard geometry. Most modern texture goniometers will give intensity $/ \phi$ plots similar to Figure 3(b) when set up correctly. The maximum slit width is determined by the size of the slit and filter holders used and has not been further optimised in the present experiments.

\section{Specimen displacement}

The specimen was carefully displaced from its correct position in the goniometer by using measured shims. The intensity at $\phi=90^{\circ}$ for the (111), (200) and (220) reflections as a function of the displacement is shown in Figure 4. The curve is quite sharply peaked at a displacement of $0.5 \mathrm{~mm}$. Reference to Figure 2 shows that the outer extremities of the beam just touch the specimen at this displacement. 


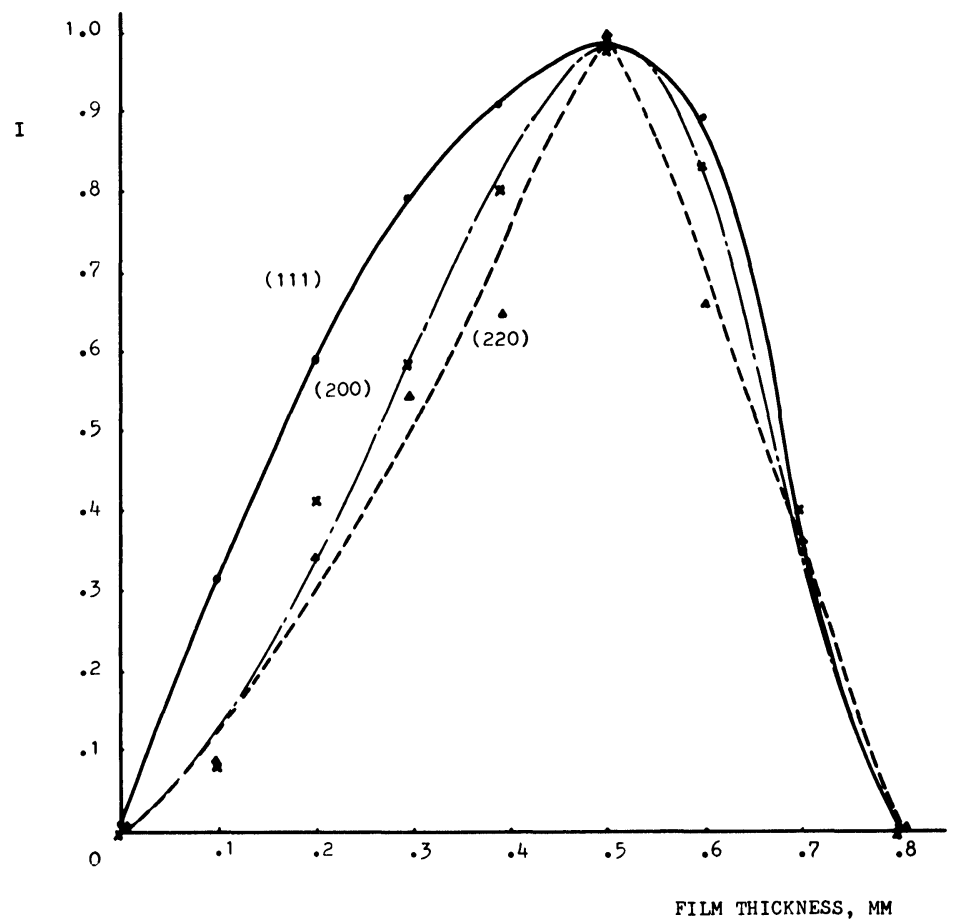

FIGURE 4 Diffracted intensity vs. sample displacement for (111), (200) and (220) reflections.

\section{Intensity variation with $\alpha$-angle}

Figure 5 shows the measured variation in intensity, with $\alpha$, at $\phi=90^{\circ}$ for the (111) reflection of a cold rolled aluminium sample. The peaks from the (110) [112] component of the texture are clearly resolved although some asymmetry is present, some of which may be attributed to the sample and the remainder to minor misalignment of the goniometer.

\section{Experimental pole figures}

Figure 6 shows the print out from the microcomputer for the (111) pole figure of a cold-rolled aluminium sample corrected for background absorption and defocusing. The intensities in this figure are normalised to give eleven equal intervals up to the maximum but alternate 


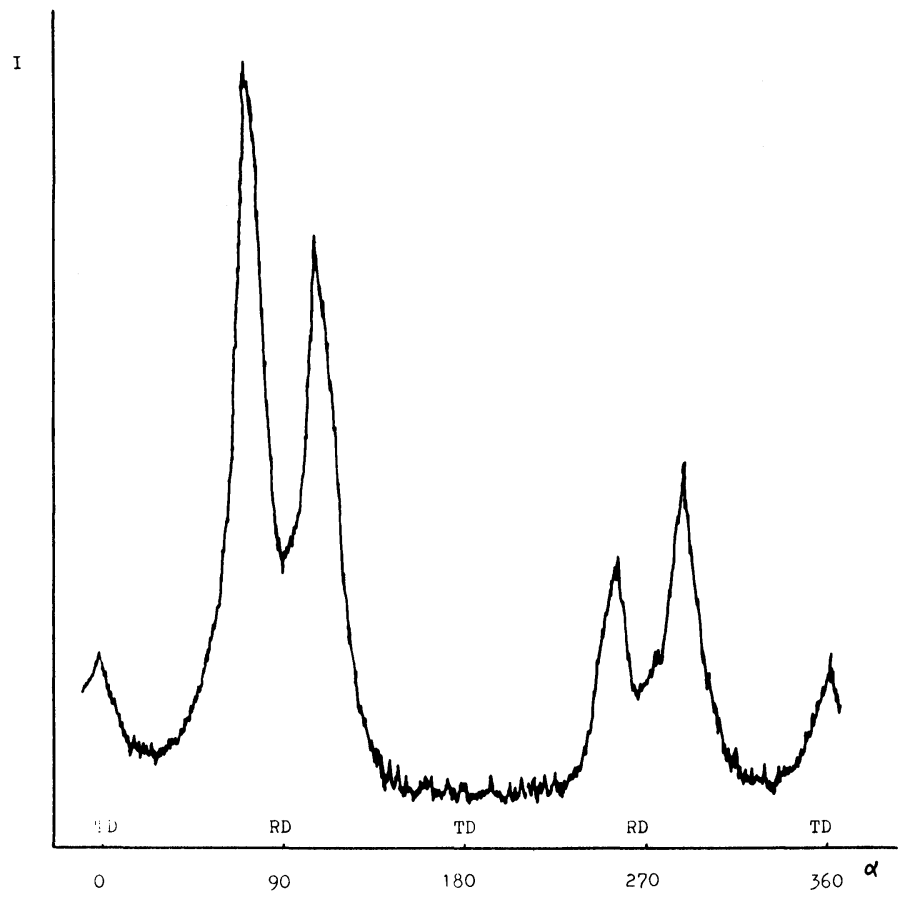

FIGURE 5 Diffracted intensity as a function of the rotation angle $\alpha$ at $\phi=90^{\circ}$ for a cold rolled aluminium sheet sample.

presentations including multiples of the random intensity are provided in the software.

The pole figure obtained using the optimum geometry is compared with that from the normal geometry and other modifications in Figure 7. Quadrant A is from the optimum geometry, quadrant B the 'normal' geometry, quadrant $\mathrm{C}$ a larger than optimum displacement and quadrant $\mathrm{D}$ a smaller than optimum displacement. It can be seen that the general features of the pole figures are retained and that significantly more information is contained in the figures at high $\phi$ angles using the modified geometry.

\section{CONCLUSION}

A method of obtaining complete pole figures using the reflection 


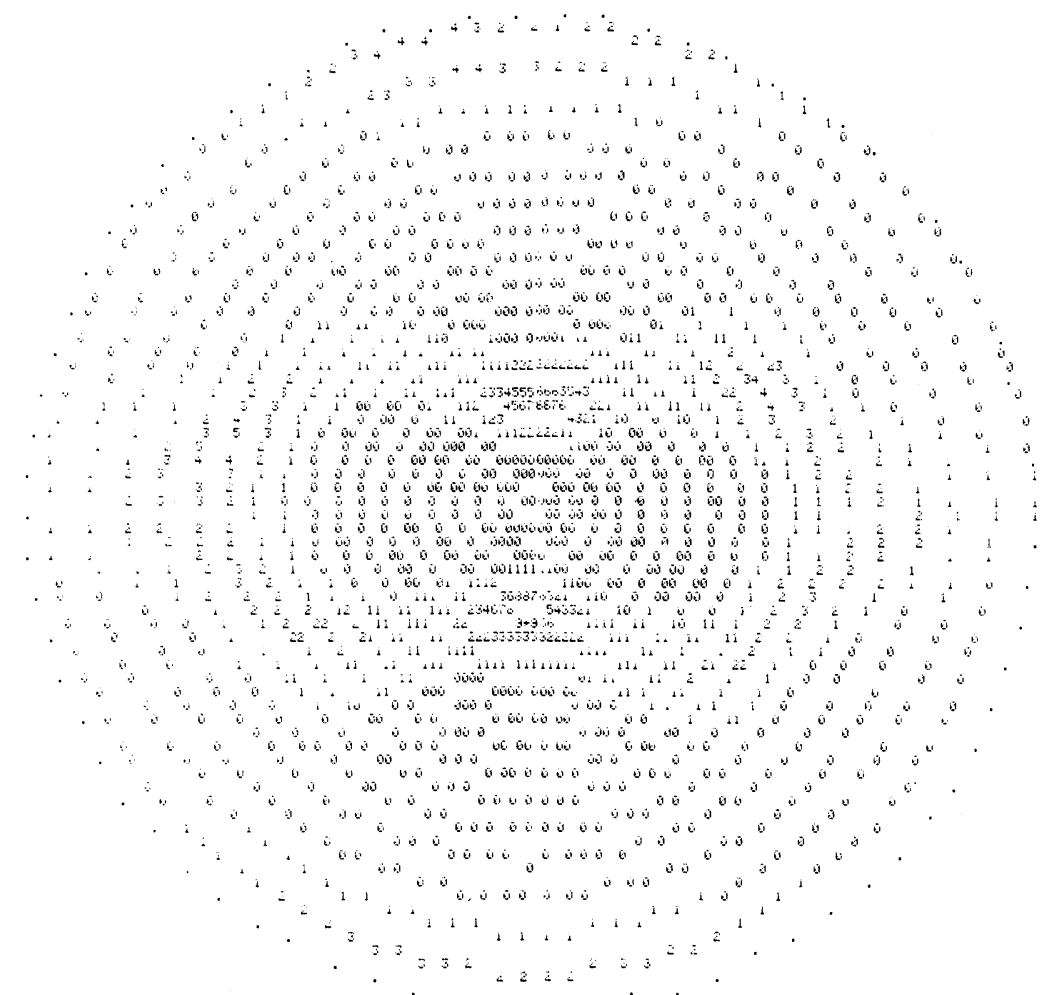

FIGURE 6 Print out of the corrected pole figure from the microcomputer.

technique alone has been described and its ability to provide greater information at high sample tilt angles has been demonstrated by experiment. Complete pole figures of sheet samples, requiring little initial preparation, can be plotted in about 2.5 hours.

\section{Acknowledgement}

One of the authors (Fan Xiong) wishes to acknowledge the receipt of a research fellowship by the Government of the Peoples Republic of China. 


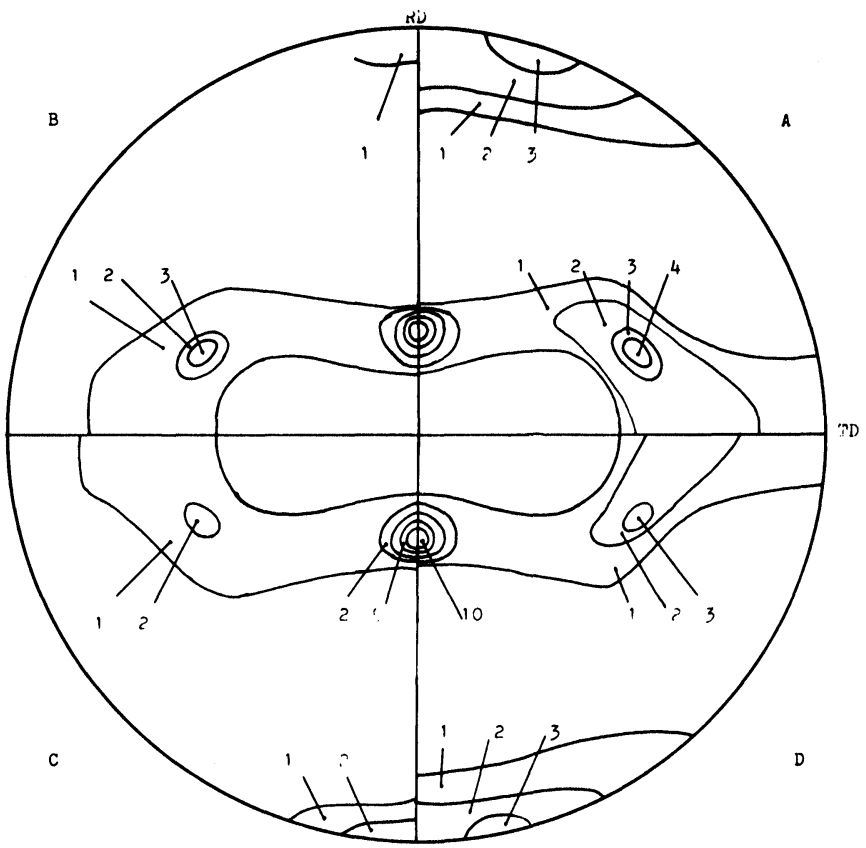

FIGURE 7 Comparison of pole figures. A. optimum geometry $(0.5 \mathrm{~mm})$; B. 'normal geometry'; C. excessive displacement $(0.8 \mathrm{~mm})$; D. inadequate displacement $(0.2 \mathrm{~mm})$.

\section{References}

Chernock, W. P. and Beck, P. A. J. Appl. Phys. 23, 341-345 (1952).

Feng, C. J. Appl. Phys. 36, 3432-3435 (1965).

Hu, H., Sperry, P. R. and Beck, P. A. Trans. AIME 194, 76-81 (1952).

Jetter, L. K. and Borie, B. S. J. Appl. Phys. 24, 532-535 (1953).

Lopata, S. L. and Kula, E. B. Trans. AIME 224, 865-866 (1962).

Ortiz, M. and Hermida, J. D. Texture Cryst. Sol. 4, 57-62 (1980).

Ortiz, M. and Hermida, J. D. Texture Cryst. Sol. 4, 159-169 (1981).

Schulz, L. G. J. Appl. Phys. 20, 1030-1033 (1949).

Tenckhoff, E. J. Appl. Phys. 41, 3944-3948 (1970). 